PROCEEDINGS OF THE

AMERICAN MATHEMATICAL SOCIETY

Volume 129, Number 4, Pages 1037-1040

S 0002-9939(00)05769-5

Article electronically published on October 11, 2000

\title{
EXISTENCE AND UNIQUENESS OF STEADY-STATE SOLUTIONS FOR AN ELECTROCHEMISTRY MODEL
}

\author{
WEIFU FANG AND KAZUFUMI ITO
}

(Communicated by David Sharp)

\begin{abstract}
We present a simple proof for the existence and uniqueness of
\end{abstract} steady-state solutions to an electrochemistry model with multiple species.

In this note we give a simple proof for the existence and uniqueness of steadystate solutions for an electrochemistry model with multiple species. The equations in such a model have been the subject of a series of papers by Choi and Lui (see 1, 2, 3, 4, and references therein). In the steady-state, the species concentrations can be expressed in terms of the electrical potential $\varphi$, and thus the problem is equivalent to solving a single integro-differential equation for $\varphi$ (see, e.g., [4] for details). Let $\Omega$ be an open, bounded $C^{0,1}$ domain in $R^{n}(n=1,2$ or 3$)$ and $\partial \Omega=\Gamma_{1} \cup \Gamma_{2} \cup \Gamma_{3}$ where $\Gamma_{1}$ and $\Gamma_{2}$ are relatively closed. Then the equation for the potential is

$$
-\epsilon \Delta \varphi=\sum_{i=1}^{m} \frac{z_{i} C_{i} e^{-z_{i} \varphi(x)}}{\int_{\Omega} e^{-z_{i} \varphi(y)} d y}+Q(x)
$$

with boundary conditions

$$
\varphi=-\alpha / 2 \text { on } \Gamma_{1}, \quad \varphi=\alpha / 2 \text { on } \Gamma_{2} \text { and } \frac{\partial \varphi}{\partial \nu}=0 \text { on } \Gamma_{3} .
$$

Here $Q(x) \in L^{2}(\Omega)$ represents a possible background charge concentration, and $\epsilon>0$ is the permittivity. The constant $C_{i}>0$ is the prescribed mass for the $i$ th species, and $z_{i}$ is its signed charge. $\alpha>0$ is the applied potential difference between the two electrodes. (For convenience, we have translated $\varphi$ by the constant $\alpha / 2$ from the formulation in [4.) The existence/uniqueness of $\varphi$ in this problem has been shown by Choi and Lui for the case $n=1$ in [1, 2, and for the case $n \geq 2$ in [3], both under the assumptions that $Q \equiv 0$ and $\sum z_{i} C_{i}=0$. These assumptions were then removed in [4]. Their arguments varied from case to case, and were quite involved, especially for the uniqueness. The proof that we will present in this note is simple and valid for all cases, and provides an explicit $L^{\infty}$ bound for the solution. In particular, our argument for uniqueness is short and elementary.

Received by the editors June 22, 1999.

2000 Mathematics Subject Classification. Primary 45K05, 35J20.

Key words and phrases. Electrochemistry, integro-differential equations.

The research of the first author was supported by Army Research Office grant DAAG55-98-10261 .

The research of the second author was supported by Air Force Office of Scientific Research grant AFOSR-F49620-95-1-0447. 
We begin with the weak form of the problem. A weak solution to (11)-(2) is defined as a function $\varphi \in H^{1}(\Omega) \cap L^{\infty}(\Omega)$ with $\varphi=-\alpha / 2$ on $\Gamma_{1}$ and $\varphi=\alpha / 2$ on $\Gamma_{2}$ that satisfies

$$
\epsilon\langle\nabla \varphi, \nabla \psi\rangle=\langle f(\varphi)+Q, \psi\rangle
$$

for all $\psi \in V=\left\{\psi \in H^{1}(\Omega): \psi=0\right.$ on $\left.\Gamma_{1} \cup \Gamma_{2}\right\}$, where we set

$$
f(\varphi)=\sum_{i=1}^{m} \frac{z_{i} C_{i} e^{-z_{i} \varphi}}{\int_{\Omega} e^{-z_{i} \varphi(y)} d y}
$$

Here $\langle\cdot, \cdot\rangle$ denotes the usual $L^{2}$ inner product on $\Omega$.

To show existence, we construct the solution map $\mathcal{T}$ as follows. For each $\tilde{\varphi} \in$ $X=\left\{\phi \in L^{2}(\Omega):|\phi(x)| \leq k\right\}(k \geq \alpha / 2$ is to be chosen below $)$, define $\varphi=\mathcal{T} \tilde{\varphi}$, where $\varphi \in H^{1}(\Omega)$ with $\varphi=-\alpha / 2$ on $\Gamma_{1}$ and $\varphi=\alpha / 2$ on $\Gamma_{2}$ is the unique solution to

$$
\epsilon\langle\nabla \varphi, \nabla \psi\rangle=\langle\tilde{f}(\varphi)+Q, \psi\rangle, \quad \psi \in V
$$

where

$$
\tilde{f}(\varphi)=\sum_{i=1}^{m} \frac{z_{i} C_{i} e^{-z_{i} \varphi}}{\int_{\Omega} e^{-z_{i} \tilde{\varphi}(y)} d y}
$$

The unique existence of such $\varphi$ is standard since $\tilde{f}(\varphi)$ is monotone decreasing in $\varphi$ and hence the unique solution $\varphi$ is the minimizer of the convex, lowersemicontinuous functional

$$
J(\varphi)=\frac{\epsilon}{2}|\nabla \varphi|_{2}^{2}+\sum_{i=1}^{m} C_{i} \frac{\int_{\Omega} e^{-z_{i} \varphi(y)} d y}{\int_{\Omega} e^{-z_{i} \tilde{\varphi}(y)} d y}-\langle Q, \varphi\rangle
$$

in a convex set of $H^{1}(\Omega)$. Clearly $\mathcal{T}$ is compact due to the compact embedding of $H^{1}$ to $L^{2}$, and it can be easily shown that $\mathcal{T}$ is continuous with respect to the $L^{2}$ norm. Next we choose $k$ so that $\mathcal{T}$ maps $X$ into itself. Notice that

$$
z_{i}|\Omega| e^{-z_{i} k} \leq z_{i} \int_{\Omega} e^{-z_{i} \tilde{\varphi}(y)} d y \leq z_{i}|\Omega| e^{z_{i} k}
$$

and thus

$$
\frac{1}{|\Omega|} \sum_{i=1}^{m} z_{i} C_{i} e^{-z_{i}(\varphi+k)} \leq \tilde{f}(\varphi) \leq \frac{1}{|\Omega|} \sum_{i=1}^{m} z_{i} C_{i} e^{-z_{i}(\varphi-k)} .
$$

Hence by setting $\psi=\varphi_{k} \equiv(\varphi-k)^{+} \in V$ in (5) we obtain

$$
\epsilon\left\langle\nabla \varphi_{k}, \nabla \varphi_{k}\right\rangle=\left\langle\tilde{f}(\varphi)+Q, \varphi_{k}\right\rangle \leq\left\langle q, \varphi_{k}\right\rangle
$$

where

$$
q(x)=\frac{1}{|\Omega|} \sum_{i=1}^{m} z_{i} C_{i}+Q(x) .
$$

Here $q$ measures the total charge concentration in the system. Under the assumptions in [1, 2, 3], $q \equiv 0$ and the usual maximum principle applies to yield $|\varphi| \leq \alpha / 2$. In the general case when $q \not \equiv 0$, we apply a more general version of the maximum 
principle, which is stated in Lemma A below. From this result, inequality (6) yields that $\varphi(x) \leq k^{*}$ for all $x \in \Omega$, where

$$
k^{*}=\frac{\alpha}{2}+\frac{4 \alpha_{6}^{2}|\Omega|^{1 / 6}}{\epsilon}|q|_{2} .
$$

Similarly, by choosing $\psi=(\varphi+k)^{-}=-(-\varphi-k)^{+} \in V$ in (5) we can show that $-\varphi(x) \leq k^{*}$. Thus, we have established the $L^{\infty}$ bounds for $\varphi=\mathcal{T} \tilde{\varphi}$ :

$$
|\varphi(x)| \leq k^{*} \text { for } x \in \Omega \text {. }
$$

Therefore, when we choose $k=k^{*}$ in the definition of $X$, the solution map $\mathcal{T}$ is from $X$ into $X$. Hence, by Schauder's fixed point theorem, $\mathcal{T}$ has a fixed point in $X$, which is a solution to (3).

As for uniqueness, it suffices to simply show that $f$ in (4) is monotone decreasing:

$$
\langle f(\varphi)-f(\hat{\varphi}), \varphi-\hat{\varphi}\rangle \leq 0
$$

for all admissible $\varphi$ and $\hat{\varphi}$. In fact each term in $f$ is monotone decreasing. To see this, let

$$
\delta_{i}=-\ln \left(\int_{\Omega} e^{-z_{i} \varphi(y)} d y\right) \quad \text { or } \quad e^{-\delta_{i}}=\int_{\Omega} e^{-z_{i} \varphi(y)} d y
$$

and similarly for $\hat{\delta}_{i}$. Then

$$
\frac{e^{-z_{i} \varphi(x)}}{\int_{\Omega} e^{-z_{i} \varphi(y)} d y}=e^{\delta_{i}-z_{i} \varphi(x)}, \quad \frac{e^{-z_{i} \hat{\varphi}(x)}}{\int_{\Omega} e^{-z_{i} \hat{\varphi}(y)} d y}=e^{\hat{\delta}_{i}-z_{i} \hat{\varphi}(x)}
$$

and

$$
\int_{\Omega} e^{\delta_{i}-z_{i} \varphi(x)} d x=\int_{\Omega} e^{\hat{\delta}_{i}-z_{i} \hat{\varphi}(x)} d x=1 .
$$

Hence $\left\langle e^{\delta_{i}-z_{i} \varphi(x)}-e^{\hat{\delta}_{i}-z_{i} \hat{\varphi}(x)}, c\right\rangle=0$ for any constant $c$. Therefore

$$
\begin{aligned}
& z_{i} C_{i}\left\langle\frac{e^{-z_{i} \varphi(x)}}{\int_{\Omega} e^{-z_{i} \varphi(y)} d y}-\frac{e^{-z_{i} \hat{\varphi}(x)}}{\int_{\Omega} e^{-z_{i} \hat{\varphi}(y)} d y}, \varphi(x)-\hat{\varphi}(x)\right\rangle \\
= & C_{i}\left\langle e^{\delta_{i}-z_{i} \varphi(x)}-e^{\hat{\delta}_{i}-z_{i} \hat{\varphi}(x)}, z_{i} \varphi(x)-z_{i} \hat{\varphi}(x)\right\rangle \\
= & C_{i}\left\langle e^{\delta_{i}-z_{i} \varphi(x)}-e^{\hat{\delta}_{i}-z_{i} \hat{\varphi}(x)},\left(z_{i} \varphi(x)-z_{i} \hat{\varphi}(x)\right)-\left(\delta_{i}-\hat{\delta}_{i}\right)\right\rangle \\
= & -C_{i}\left\langle e^{\delta_{i}-z_{i} \varphi(x)}-e^{\hat{\delta}_{i}-z_{i} \hat{\varphi}(x)},\left(\delta_{i}-z_{i} \varphi(x)\right)-\left(\hat{\delta}_{i}-z_{i} \hat{\varphi}(x)\right)\right\rangle \\
\leq & 0
\end{aligned}
$$

since the exponential function is increasing and $C_{i}>0$. Therefore $f$ is monotone decreasing in $\varphi$ and thus we easily obtain the uniqueness of weak solutions.

We complete our presentation by proving the lemma that is used to establish the $L^{\infty}$ bounds (9). We denote by $\alpha_{6}$ the constant in the Poincaré inequality $|\psi|_{6} \leq \alpha_{6}|\nabla \psi|_{2}$ for all $\psi \in V$.

Lemma A. Suppose $\phi \in H^{1}(\Omega)$, and $\phi_{\tau}=(\phi-\tau)^{+} \in V$ for $\tau \geq \bar{\tau}$. If $\phi_{\tau}$ satisfies

$$
\left\langle\nabla \phi_{\tau}, \nabla \phi_{\tau}\right\rangle \leq\left\langle F, \phi_{\tau}\right\rangle
$$

for some $F \in L^{2}(\Omega)$, then

$$
\phi(x) \leq \bar{\tau}+4 \alpha_{6}^{2}|\Omega|^{1 / 6}|F|_{2} .
$$


Proof. Let $\Omega_{\tau}=\{x \in \Omega: \phi(x)>\tau\}$. By the Poincaré and the Hölder inequalities,

$$
\alpha_{6}^{-2}\left|\phi_{\tau}\right|_{6}^{2} \leq\left|\nabla \phi_{\tau}\right|_{2}^{2} \leq\left\langle F, \phi_{\tau}\right\rangle \leq|F|_{2}\left|\phi_{\tau}\right|_{6}\left|\Omega_{\tau}\right|^{1 / 3},
$$

that is,

$$
\left|\phi_{\tau}\right|_{6} \leq \alpha_{6}^{2}|F|_{2}\left|\Omega_{\tau}\right|^{1 / 3} .
$$

For $\hat{\tau}>\tau \geq \bar{\tau}$

$$
\left|\phi_{\tau}\right|_{6}^{6} \geq \int_{\Omega_{\hat{\tau}}}\left|\phi_{\tau}\right|^{6} d x \geq(\hat{\tau}-\tau)^{6}\left|\Omega_{\hat{\tau}}\right| .
$$

Therefore

$$
\left|\Omega_{\hat{\tau}}\right| \leq\left(\alpha_{6}^{2}|F|_{2}\right)^{6}(\hat{\tau}-\tau)^{-6}\left|\Omega_{\tau}\right|^{2} .
$$

Then by applying an elementary lemma (see [5, Lemma 2.9]) to the non-increasing function $\left|\Omega_{\tau}\right|$ of $\tau$, we obtain

$$
\left|\Omega_{\tau}\right|=0 \text { for } \tau \geq \tau^{*},
$$

where

$$
\tau^{*}=\bar{\tau}+4 \alpha_{6}^{2}|\Omega|^{1 / 6}|F|_{2} .
$$

Therefore we have $\phi_{\tau^{*}}=0$ a.e. in $\Omega$. Hence $\phi \leq \tau^{*}$ as stated.

\section{REFERENCES}

[1] Y. S. Choi and R. Lui, Analysis of an electrochemistry model with zero-flux boundary conditions, Applicable Analysis, 49(1993), pp. 277-288. MR 96d:80005

[2] Y. S. Choi and R. Lui, Uniqueness of steady-state solutions for an electrochemical model with multiple species, J. Differential Equations, 108(1994), pp. 424-437. MR 95j:35225

[3] Y. S. Choi and R. Lui, Multi-dimensional electrochemistry model, Arch. Rational Mech. Anal., 130(1995), pp. 315-342. MR 96f:80007

[4] Y. S. Choi and R. Lui, An integro-differential equation arising from an electrochemistry model, Quart. Appl. Math., 55(1997), pp. 677-686. MR 99h:45017

[5] G. M. Troianiello, Elliptic Differential Equations and Obstacle Problems, Plenum Press, New York, 1987. MR 92b:35004

Department of Mathematics, West Virginia University, Morgantown, West Virginia 26506

E-mail address: wfang@math.wvu.edu

Department of Mathematics, North Carolina State University, Raleigh, North CarOLINA 27695

E-mail address: kito@eos.ncsu.edu 